\title{
The Differences of People's Social Emotions During the Initial Stage Of COVID-19 Pandemic
}

\author{
Zuozhou Huang, Guifang Fu* \\ Department of Applied Psychology, Guangdong University of Foreign Studies, Canton 510006, China \\ *Corresponding Authors: Guifang Fu, Department of Applied Psychology, Guangdong University of \\ Foreign Studies, Canton 510006, China
}

\begin{abstract}
Since the outbreak of COVID-19, people's lives and production have been affected to varying degrees, and their social emotions have also fluctuated. In order to investigate the main social emotional status and demographic differences of the public during the initial period of COVID-19, an online survey was conducted on the social emotional status of 1665 people using a self-compiled scale. The results show that: In the early stage of COVID-19, people's overall social mood, living conditions, mood at home, mood at work/study and overall social mood satisfaction were all above the medium level. There was no significant gender difference, and there were significant differences in the degree of affected by the epidemic in the areas where people lived. There are significant differences among people of different occupations in the degree of being affected by the epidemic, the degree of optimism about the epidemic, the degree of helplessness about the epidemic, the psychology of fluke and the psychology of avoidance. ; (2) In the early stage of the epidemic, the top three types of social emotions were calm, optimism and worry. People are moderately affected by the novel coronavirus, with a high level of optimism, but a low sense of helplessness, and a moderate level of fluke and avoidance. This study provides a timely understanding of people's social emotions at the initial stage of the COVID-19 pandemic, providing data support for grasping and controlling people's social emotions during the epidemic period, which is of important practical value. Key words
\end{abstract}

Key words: COVID-19; Social emotion; Social mentality; Anxiety; Depressed mood.

\section{INTRODUCTION}

Since December 2019, the outbreak of novel coronavirus has had a lasting and significant impact on people all over the world. Especially in the early stages of the outbreak, the epidemic prevention policies and public sentiment of countries around the world also varied greatly. Because the outbreak of the new champions league has sudden, universality, urgency, explosive and uncertainty, and other properties, and there was no specific methods such as drugs or vaccines can undertake the treatment and prevention, has drawn great attention of the world health organization and the countries, such as China and its cities based on the seriousness of the epidemic situation, major public health emergencies are up level response mechanism. In general, governments of all countries have taken various measures to combat the epidemic to ensure people's physical and mental health and avoid excessive panic, anxiety and other negative emotions. However, with the rapid development of the epidemic, more and more people are gradually aware of the seriousness of the disease. Due to this sudden public health event, people still appear various bad social emotions to some extent, such as fear of travel, fear of being infected by the virus, fear and excessive attention to the epidemic situation. Bad social emotions serious, even become irritable, apathy, and even have aggressive behavior or revenge thoughts.

This outbreak is a major public health event and a systemic crisis affecting the whole country with global impact. Therefore, unlike normal natural disasters, typical anxiety and anger are likely to occur during and after an epidemic, which can be divided into three stages: the incubation stage, the release stage and the awareness stage (Chen, 2013).

This study investigated the social emotions of the epidemic population at the early stage. On the one hand, the government decided how to implement epidemic prevention and control work needs to take social emotions as a reference; on the other hand, social emotions need correct guidance, which is 
conducive to epidemic prevention and control.

Social emotion is a complex and relatively stable attitude experience formed by people's perception of various situations in social communication and life and through the interaction and effect between group members. It is a barometer of social operation (Fu, 2013). Social emotion refers to people's perception of various situations in social life and the relatively complex and stable attitude experience formed through the mutual influence and interaction among group members. Such perception and experience have a guiding and dynamic influence on individuals or the whole (Sha, Feng,2006). Wang JunXiu (2013) believes that from the perspective of social mentality, social emotion is defined as the universal and consistent psychological characteristics and behavioral patterns of most members of the society formed under the influence of social environment and culture in a certain period, and it becomes the behavioral template of each individual member. He believes that social emotion is the emotional experience shared by most members of a group and society. Liang Yan (2006) believes that social emotion is not only a subjective indicator of social members' own living conditions, but also a subjective indicator of social operation and social contradictions. It is a social reflection based on individuals' cognition and psychological changes of people and things in the society. Li Ruisheng (2008) advocated that social emotions should be investigated based on specific social and cultural backgrounds. He believed that "once individual emotions are socialized, individual emotions will be externalized into collective emotions with a common psychological orientation and characteristics, which is social emotions. Li Huilong (2019) divided the understanding of social emotions into two specific levels -- individual level and more macroscopic group level, namely, individual social emotions and group social emotions. Individual social emotions focus on the socialization of individual emotions, while group social emotions focus on the interaction between group members. There is an inseparable relationship between the two, which together constitute the two basic dimensions of social emotion and help people better understand the connotation of social emotion.

In the existing literature, domestic researchers have not reached a consensus on the concept of social emotion, so there is no clear and unified definition. The research on social emotion needs to be further studied. Although previous studies have also involved the psychological state of people in emergencies, most of the research objects are college students, and the group targeted is not comprehensive enough, and the research on panic emotion of people in emergencies is rare. This study investigated and analyzed the difference of people's social emotions during the epidemic period, which can provide a basis for social psychological services during the epidemic period and help the society improve the psychological intervention mechanism.

\section{METHOD}

\subsection{Subject}

The subjects of this study are people in all parts of China (mainly in Guangdong province). The questionnaire on Psychological State during the COVID-19 outbreak was released on the Wenjuanxing and distributed through Wechat, and 1,665 valid questionnaires were recovered, with an availability rate of $97 \%$. The specific distribution of gender, age, province, place of residence and occupation of the subjects: the subjects are mainly female, 18-25 years old, mainly in Guangdong province, mainly in cities, and mainly college students. The overall structure is well distributed, which meets the requirements of being a subject.

\subsection{Tools}

By referring to the questionnaire designed by Zhou Xin, Wu Yuting and Wu Zhenghu (2009) and the relevant questionnaires already published on the Internet, and selecting some items from them as the item sources of some scales, the social emotion scale was compiled by ourselves, including the basic status of social emotion and three subscales. The basic social mood survey mainly includes people's general mood and the degree of influence. For example, people's general mood includes five questions. The first four questions are: How is your general mood during the epidemic? How do you feel about your life during the epidemic? How is your mood at home during the epidemic? How did you feel in your work or study during the epidemic? The first four questions are graded on a Likert scale of 5, where 1 means very good, 2 means very good, 3 means fair, 4 means not very good, and 5 means very bad. The last topic is what is your main emotion during the epidemic? The alternative 
answers were happy, excited, calm, optimistic, sad, angry, feeling nothing, sad, afraid, worried, helpless, and anxious.

The three subscales were anxiety scale, with a total of 4 questions, and its internal consistency coefficient was 0.735 Panic scale, a total of 3 , its internal consistency coefficient was 0.679 ; Depression scale, a total of 5 questions, its internal consistency coefficient was 0.775 The three subscales were based on Likert rating criteria, and were divided into five levels: 1 very inconsistent, 2 relatively inconsistent, 3 uncertain, 4 relatively consistent, and 5 very consistent. The internal consistency coefficient of the self-made scale was 0.907 .

\subsection{Data Statistics}

SPSS 24.0 software was used to analyze the survey data, mainly using descriptive statistics, difference test and other statistical methods.

\section{RESUlts}

\subsection{Basic Situation of People's Social Emotions During the Epidemic}

\subsubsection{People's overall mood}

Descriptive statistics and single-sample $\mathrm{T}$ test are used to analyze the satisfaction of the subjects' general mood, living status, mood at home, mood at work/study and overall social mood. The results are shown in Table 1.

Table1. The general social mood of the people at the initial stage of the epidemic

\begin{tabular}{|c|c|c|c|c|}
\hline The title & $\mathrm{M}$ & $\mathrm{SD}$ & $\mathrm{t}$ & $\mathrm{p}$ \\
\hline 1. How is your general mood during the epidemic? & 3.53 & .908 & -21.021 & $.000^{* * * *}$ \\
\hline 2. How do you feel about your life during the epidemic? & 3.45 & .932 & -23.908 & $.000^{* * * *}$ \\
\hline 3. How did you feel at home during the outbreak? & 3.55 & .873 & -20.837 & $.000^{* * * *}$ \\
\hline 4. How did you feel in your work or study during the epidemic? & 3.31 & 1.000 & -28.026 & $.000^{* * * *}$ \\
\hline 5. Overall social emotions during the epidemic period & 3.46 & .817 & -26.811 & $.000^{* * * *}$ \\
\hline
\end{tabular}

****p<.001 Notes: The total social mood during the epidemic period is the sum of questions 1-4

According to Table 1, there were significant differences between the satisfaction of subjects' general mood, living status, home mood, work/study mood and general social mood and the score 4 representing "satisfaction" (P\&LT;.001), indicating that the subjects' satisfaction in general mood, living conditions, mood at home, work/study mood and general social mood during the epidemic period was above the average level.

Table2. Basic information of people's affected degree, optimism degree, helplessness degree, fluke psychology and avoidance psychology

\begin{tabular}{|c|c|c|}
\hline The title & M & SD \\
\hline 1. The extent of the impact of COVID-19 & 2.53 & 1.148 \\
\hline 2. Optimism about COVID-19 & 3.77 & .934 \\
\hline 3. Helplessness over COVID-19 & 2.17 & 1.035 \\
\hline 4. Chance & 3.12 & .930 \\
\hline 5.avoidance & 3.42 & .514 \\
\hline
\end{tabular}

From Table 2 and Figure 1, it can be seen that the specific conditions of the subjects' answers about the degree of being affected by the epidemic, the degree of optimism about the epidemic, the degree of helplessness about the epidemic, the fluky psychology and the avoidance psychology. It can be seen from the table 2 that people are moderately affected by COVID-19, with a high level of optimism and a low sense of helplessness, and a moderate level of fluke and avoidance psychology. Nearly half of the subjects answered that the epidemic had a great impact on them, more than half of the subjects were optimistic about the epidemic, more than half of the subjects had a low sense of helplessness, 
most of the subjects would have a fluky mind, and nearly half of the subjects were not sure whether they would have the avoidance psychology.

\subsubsection{The Main Types of Social Emotions in the Initial Stage Of COVID-19}

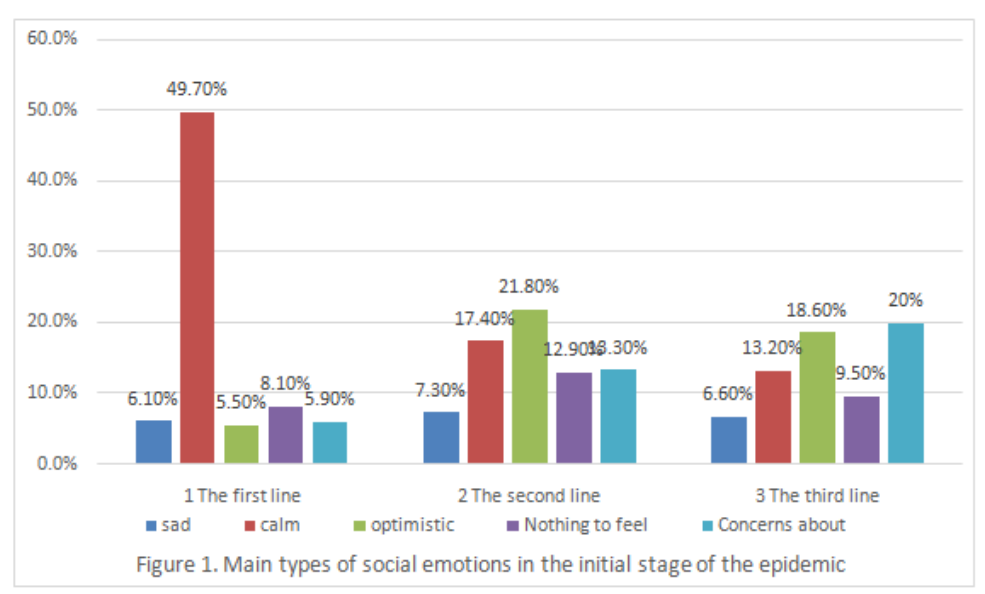

Figure 1 shows the specific situation of people's major emotional types during the epidemic. It can be seen from the table that the first emotion type is mainly calm, the second emotion type is mainly optimistic and calm, and the third emotion type is mainly worry, optimism and calm. It can be seen that the top three emotion types of people during the epidemic are calm, optimism and worry.

\subsection{Analysis of Differences in Social Emotions}

The difference test was used to analyze the difference of people's affected degree, optimism degree, helplessness degree, luck psychology and avoidance psychology by gender, region and occupation respectively. The results showed that gender difference was not significant, and there were significant differences in the affected degree of people in the area. There are significant differences among people of different occupations in the degree of being affected by the epidemic, the degree of optimism towards the epidemic, the degree of helplessness towards the epidemic, the psychology of fluke and the psychology of avoidance.

Table 3 reports the results of an OVA for each factor in terms of living environment (community presence/absence/unknown cases). According to the analysis results, there was no significant difference between anxiety and depression in living environment (with/without/unknown cases in the community).There were significant differences in panic levels in the living environment (with/without/unknown cases in the community) .Post-mortem analysis showed that the difference in panic degree in living environment (community with/without/unknown cases). The mean difference between those who had no cases and those who had cases in their communities was -.188, $\mathrm{p}=.027$. That is to say, participants with cases in their communities had significantly higher average levels of panic than participants without cases in their communities.

Table3. Analysis of variance between main variables and living environment (community with/without/unknown cases)

\begin{tabular}{|l|l|l|l|l|l|l|}
\hline factors & The living environment & $\mathrm{N}$ & $\mathrm{M}$ & $\mathrm{SD}$ & Homogeneity test & $\mathrm{F}$ \\
\hline \multirow{4}{*}{ anxiety } & No cases of & 1400 & 2.48 & .863 & .569 & 1.642 \\
\cline { 2 - 7 } & There are cases & 187 & 2.60 & .879 & & \\
\cline { 2 - 7 } & Don't know & 78 & 2.55 & .835 & & \\
\hline \multirow{5}{*}{ panic } & No cases of & 1400 & 2.59 & .865 & .421 & $3.906^{*}$ \\
\cline { 2 - 7 } & There are cases & 187 & 2.78 & .923 & & \\
\cline { 2 - 7 } & Don't know & 78 & 2.64 & .821 & & \\
\hline \multirow{5}{*}{ depression } & No cases of & 1400 & 2.00 & .756 & .588 & \\
\cline { 2 - 7 } & There are cases & 187 & 2.08 & .787 & & \\
\cline { 2 - 7 } & Don't know & 78 & 2.08 & .718 & & \\
\hline
\end{tabular}

$* p<.05$

\section{DisCUSSIONS}

\subsection{Basic Situation of People's Social Emotions During the Epidemic}


According to the survey of people's psychological state during the COVID-19 outbreak, people's general mood, living conditions, mood at home, work/study mood and overall social mood satisfaction are all above the average level, indicating that people's general mood has not been greatly affected. First, there is no significant gender difference, indicating that there is no significant difference in general social mood between men and women. Second, there are significant differences in the extent of the impact of the epidemic in the areas where people live. To be specific, compared with people from Guangdong province, people from non-Guangdong province think they are more affected by the epidemic. This may be because Guangdong province is geographically far away from Wuhan, which was severely affected by the epidemic. Third. And the degree of the identity of the people in the affected by the outbreak, the outbreak of optimism's helplessness in the outbreak, fluky psychology and avoid psychological significant differences are, in particular, relative to the university students, college students think that the outbreak of the influence of their smaller, to feel more optimistic, new crown outbreak of epidemic situation of helplessness is lower and less likely to produce by luck and avoid psychological; Fourth, the top three social emotions during the epidemic period were calm, optimistic and worried, indicating that most people's mood fluctuated little and were optimistic, but still worried about the epidemic.

On the whole, people are moderately affected by the novel coronavirus, with a high degree of optimism and a low sense of helplessness, while people's fluke and avoidance psychology are at a medium level.

\subsection{The Presence or Absence of Cases in the Community is the Main Reason Affecting Social Emotions}

It can be seen from the structural model of influencing factors of panic emotion that the living environment is not the direct cause of panic emotion whether it is people at home or at work or people with or without cases in their community. Even so, the presence or absence of cases in the community in which people live can influence their social mood. Specifically, people in their communities with cases are more suspicious and fearful than those in their communities without cases.

\subsection{Analysis of Differences in Social Emotions}

Panic is produced by the mediating effect of doubt, anxiety and interpersonal trust crisis. The results show that: 1) People in different regions think that the epidemic has a different impact on them. People in Guangdong province think that the epidemic has less impact on them because they are a certain distance away from Wuhan, the epicenter of the outbreak.2) The identity of the people also has an impact on their social emotions. College students think that the epidemic has a greater impact on them, but they feel more optimistic about the epidemic.3) People's living environment has a serious impact on their social emotions, and people who work are more likely to have doubts and panic.

\subsection{Understand the Law of Development of Psychological Stress}

In order to implement effective psychological intervention, the first step is to understand the rules of psychological crisis. When people experience irregular emergencies, they may experience psychological stress, which can be divided into four stages, respectively: First, the alert response period: this period of people's performance is high alert, panic, bewildered or a blank head; The second, Negative defense period: In order to control bad emotions, individuals instinctively start their own defense mechanisms, such as avoidance, denial, anxiety, depression, emotional expression control or choose to ignore the existence of danger; The third, Reality adaptation period: After the crisis occurs for a period of time, people can take a positive attitude to face and accept the reality, and seek a variety of methods to solve the current problems, so as to gradually reduce their anxiety and improve their social functions; Recovery period: Most people grow up after a crisis, become more rational and mature psychologically and behaviourally, and begin to acquire positive coping strategies in certain ways. It is necessary to understand the main manifestations of the epidemic psychological crisis. During the epidemic, people may have excessive psychological reactions in cognitive, emotional, behavioral and physical aspects. For example, symptoms such as inattention, anxiety and panic, not going out for a long time during the epidemic, feeling panic, headache and loss of appetite. That is to help people in crisis to clarify the essence of psychological problems, with a more correct and effective way to deal with the crisis, help rebuild confidence, give full play to their own ability and potential, restore psychological balance and normal growth and adapt to the process of life. 


\section{CONCLUSION}

The findings of this study are as follows:(1) In the early stage of COVID-19, people's overall social mood, living conditions, mood at home, mood at work/study and overall social mood satisfaction are above the average level. There is no significant gender difference, and there are significant differences in the degree of affected by the epidemic in the areas where people live. There were significant differences in occupational impact, optimism, helplessness, fluke and avoidance. (2) The top three types of social emotions during the epidemic were calm, optimism and worry; People are moderately affected by the novel coronavirus, with a high level of optimism, but a low sense of helplessness, and a moderate level of fluke and avoidance.

\section{LIMITATIONS}

First of all, the self-made scale in this paper has limitations. Since there is no ready-made scale, I divided multiple dimensions through other similar questionnaires, and selected some appropriate questions as part of the scale, resulting in uneven distribution of questions in all dimensions and low validity of some dimensions. Secondly, this paper adopts the questionnaire method to conduct the investigation. When the subjects answer the questionnaire, it is difficult to exclude the effects of defense mechanism, random filling and other factors, which affect the accuracy of the research results. The interview method can be considered for future research. The research error can be reduced to some extent, and more in-depth information can be obtained. In addition, due to the limited resources, the questionnaire participants are mainly college students with a limited age range and cannot represent the majority of the Chinese population. Finally, due to my limited ability, I have not explored many problems deeply enough, and there are certain limitations. I hope that further discussion and correction can be made in future studies.

\section{REFERENCES}

[1] National Health Commission. Notice on the issuance of guidelines for emergency psychological crisis intervention in novel Coronavirus pneumonia Outbreak. [EB/OL].[2020-01-27]. http://www.nhc.gov.cn/ xcs/zhengcwj/202001/6adc08b966594253b2b791be5c3b9467.shtm

[2] Chen Manqi. (2013). Group sentiment and its measurement. Social Science Front (02), 182-187.

[3] Du Zhiliang. Evaluation and cultivation of urban residents' social mentality, Master's thesis. Guangdong University of Foreign Studies, 2018.

[4] Guifang Fu. On the formation mechanism of the new generation of migrant workers' social mentality [J].search, 2013 (06):243-245.

[5] Liang Yan. Thoughts on the establishment of social emotional counseling mechanism. Journal of Armed Police Academy (1), 84-86.

[6] Li Ruisheng. (2008). Introduction to social Emotions. Social Science Forum: Academic Research volume, 000(004), P.60-63.

[7] Li Huilong. Study on social emotional governance in government public opinion [D]. Jilin university,2019.

[8] Mohammed A. Mamun, Mark D. Griffiths. First COVID-19 suicide case in Bangladesh due to fear of COVID-19 and xenophobia: Possible suicide prevention strategies [J]. Asian Journal of Psychiatry, 2020, 51.

[9] Sha Lian-xiang, Feng Bolin, et al. Social psychology [M].Bei Jing: China Renmin University Press,2006.

[10] Wang Junxiu. Theory of Social Psychology: a macroscopic paradigm of social psychology [M]. Bei Jing: Social Sciences Academic Press, 2014.

[11] Wang Junxiu. (2013). Attention to social emotions to promote social identity and social consensus-A Study on Chinese social mentality (2012 2013).Democracy and Science (1), 64-71.

[12] Yang Maolin. Research on the influencing mechanism of group self-esteem of migrant workers from the perspective of cultivating positive social mentality, Master's thesis. Guangdong University of Foreign Studies, 2019.

[13] Zhou Xin, Wu Yuting, Wu Zhenghu. Study on the psychological state and intervention mechanism of college students during the influenza A (H1N1) epidemic period[J].Health research,2009,29(05):351-354

Citation: Zuozhou Huang, Guifang Fu. "The Differences of People's Social Emotions During the Initial Stage Of COVID-19 Pandemic" International Journal of Managerial Studies and Research (IJMSR), vol 9, no. 8, 2021, pp. 48-53. doi: https://doi.org/10.20431/2349-0349.0908006.

Copyright: (C) 2021 Authors. This is an open-access article distributed under the terms of the Creative Commons Attribution License, which permits unrestricted use, distribution, and reproduction in any medium, provided the original author and source are credited. 